\title{
Nomenclature TARCO approuvée par le Cockpit
}

\author{
Christian Oeschger ${ }^{a}$, Patrick Müller ${ }^{b}$
}

a Expert, division Médecine et tarifs ambulatoires; ${ }^{b}$ Chef de la division Médecine et tarifs ambulatoires

La sixième séance du Cockpit, l’organe de pilotage du projet TARCO, s'est déroulée le 29 novembre 2017. A cette occasion, les délégués du Cockpit ont approuvé la version finale de la nomenclature quasi complète de la structure tarifaire "TARCO» révisée par la $\mathrm{FMH}$. La décision a été précédée de neuf mois d'intenses travaux, durant lesquels les experts de la FMH ont révisé, en collaboration avec toutes les sociétés de discipline affiliées, la structure tarifaire rejetée lors de la votation générale de 2016.

Après le rejet de la proposition de révision du tarif médical ambulatoire par la majorité des membres votants de la FMH en juin 2016, l'Assemblée des délégués a chargé en septembre 2016 le Comité central et la division Médecine et tarifs ambulatoires de poursuivre la révision tarifaire. En février 2017, les travaux ont été lancés avec le concours de toutes les sociétés de discipline dans le cadre du projet TARCO (=TARMED consensus).

Les experts de la FMH ont révisé les différents chapitres dans plus de 30 groupes de travail, en collaboration avec les "sociétés de discipline leaders" préalablement désignées dans le cadre d'une consultation auprès de toutes les sociétés de discipline. Les médecins ont ensuite révisé la structure des chapitres avec l'appui des experts tarifaires, mais surtout une fois de plus étudié en détail les différentes positions tarifaires: dans d'innombrables séances et e-mails, les minutages ont encore une fois été révisés, les interprétations médicales complétées et reformulées, et les "valeurs intrinsèques qualitatives» réintroduites et fixées pour toutes les positions tarifaires. Contrairement au précédent projet, chaque chapitre a ensuite été mis en consultation auprès des autres sociétés de discipline pendant six semaines. Les 500 retours ont été transmis aux groupes de travail concernés. Tous ces retours ont été analysés et, là où cela semblait approprié, intégrés. Ensuite, les sociétés de discipline ont été informées des raisons de la non-prise en compte de certains retours. En procédant de la sorte, le groupe d'experts est parvenu au consensus le plus large possible parmi toutes les sociétés de discipline impliquées.

\section{Le Cockpit a fait ses preuves}

La structure de l'organisation a également contribué de manière décisive à la réussite du projet. Pour la première fois et contrairement au précédent projet, la FMH a mis en place, avec le Cockpit, un organe de pilotage largement appuyé par toutes les organisations de la FMH. Les organisations suivantes sont représentées dans le Cockpit:

- Verband Deutschschweizerischer Ärztegesellschaften (VEDAG, 1 siège)

- Société Médicale de la Suisse Romande (SMSR, 1 siège)

- Ordine dei Medici del Cantone Ticino (OMCT, 1 siège)

- Foederatio Medicorum Psychiatricorum et Psychotherapeuticorum (FMPP, 2 sièges)

- Foederatio Medicorum Chirurgicorum Helvetica (FMCH, 2 sièges)

- Collège de médecine de premier recours (CMPR, 2 sièges)

- Swiss Federation of Specialities in Medicine (SFSM, 2 sièges)

- Association des médecins dirigeants d'hôpitaux de Suisse (AMDHS, 2 sièges)

- Association suisse des médecins indépendants travaillant en cliniques privées et hôpitaux (ASMI, 1 siège, détails voir ci-après)

L'Association suisse des médecins-assistant(e)s et chef(fe)s de clinique (ASMAC, 2 sièges) était également invitée. Les sociétés de discipline médicale reconnues (annexe 2 des Statuts de la FMH) qui ne sont affiliées à aucune association faîtière, mais qui sont, d'après les 


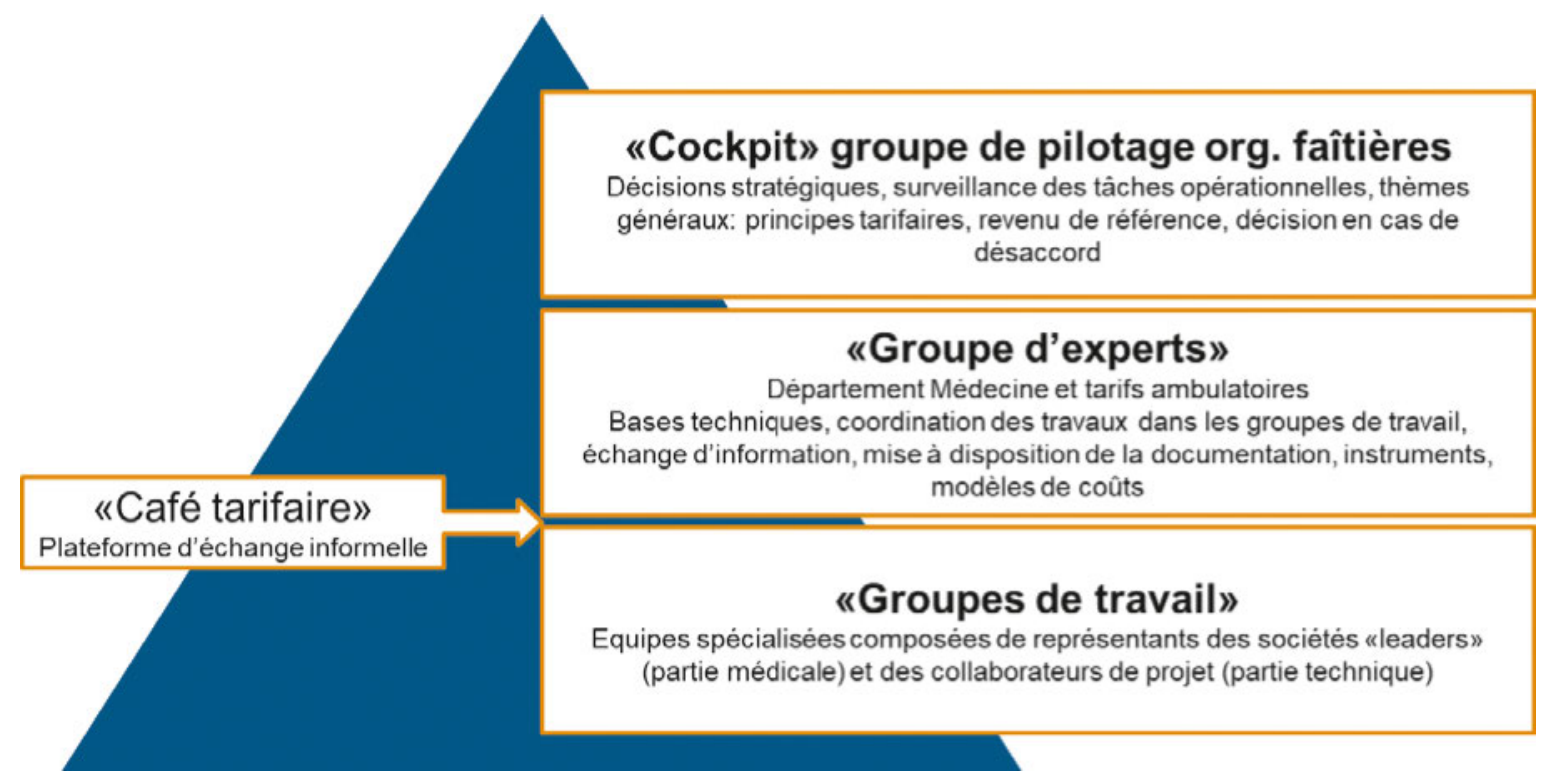

Organisation du projet TARCO.

Statuts de la FMH, autorisées à intervenir (annexe 3) sont représentées par l'Association suisse des médecins indépendants travaillant en cliniques privées et hôpitaux (1 siège). Les sociétés concernées s'étaient préalablement mises d'accord à ce sujet.

Lors des nombreuses séances tenues au cours des derniers mois, les délégués du Cockpit ont à maintes reprises discuté différentes propositions des groupes de travail ou sociétés de discipline et pris au total plus de 100 décisions. Celles-ci concernaient les principes tarifaires, la nomenclature et les «valeurs intrinsèques qualitatives».

\section{La version finale de la nomenclature est là}

Lors de la sixième séance du Cockpit à la fin novembre 2017, le groupe d'experts a soumis l'ensemble des positions tarifaires, à l'exception de deux chapitres ("Appareil locomoteur et chirurgie de la main» et "Prestations générales en salle d'opération»), à l'approbation des délégués. Par rapport à la version qui avait échoué en votation générale, un grand nombre de chapitres ont une nouvelle fois été révisés. En conséquence, le nombre total de positions a encore légèrement augmenté (2700 positions), mais reste malgré tout nettement inférieur au TARMED en vigueur (actuellement environ 4500 positions). De plus, le tarif inclut désormais aussi des positions tarifaires spécifiques dans les domaines "Génétique médicale», "Gériatrie», «Médecine hyperbare», "Infectiologie», "Néphrologie» et «Oncologie médicale et hématologie». Le Cockpit a approuvé l'ensemble des chapitres soumis et donné au groupe d'experts de la FMH le mandat de négocier la structure tarifaire avec les partenaires tarifaires. Les deux chapitres qui restent à finaliser seront probablement approuvés lors de la séance du Cockpit du 10 janvier 2018 et aussi intégrés dans les négociations.

\section{Les négociations avec les partenaires tarifaires ont déjà commencé}

$\mathrm{Au}$ sein de l'ats-tms SA, les discussions au niveau des modèles de coûts ont déjà débuté. La FMH et ses fournisseurs de données pour calculer les taux de coûts - la Caisse des médecins et les sociétés cantonales de médecine ainsi que NewIndex SA - sont invités à établir davantage de transparence pour le modèle de coûts KOREG. D'intenses discussions sont actuellement en cours pour assurer cette transparence, car les données sont d'une grande importance pour la FMH et ses membres. L'essentiel est que la FMH ne fournira pas de données individuelles et que seul le secrétariat de l'atstms SA pourra consulter les données agrégées. Les associés / partenaires tarifaires n'y auront par contre pas accès. De plus, l'examen des données brutes ne pourra s'effectuer que par une fiduciaire indépendante.

Eu égard aux nombreuses réactions des sociétés de discipline, la FMH a demandé aux partenaires tarifaires $\mathrm{H}+$, curafutura et CTM de vérifier encore une fois le modèle de coûts INFRA. Les experts ont décidé que la vérification ne portera pas sur tous les paramètres des unités fonctionnelles, ni sur toutes les unités fonctionnelles INFRA. Elle concernera en revanche toutes les unités fonctionnelles qui génèrent un volume de 
Correspondance: FMH / division Médecine et tarifs ambulatoires

Baslerstrasse 47 CH-4600 Olten

Tél. 0313591230 Fax 0313591238 tarife.ambulant[at]fmh.ch points tarifaires important ou pour lesquelles les frais de personnel et d'infrastructure sont très élevés. Par ailleurs, toutes les unités fonctionnelles qui présentent une augmentation disproportionnée des coûts par rapport aux taux de coûts de la version $1.08 .00 \mathrm{du}$ TARMED seront également encore une fois contrôlées. Si les paramètres d'une unité fonctionnelle sont une nouvelle fois vérifiés, cet examen portera aussi sur les «unités fonctionnelles voisines» afin de garantir une vue d'ensemble.

\section{Calendrier}

Dans les semaines à venir, la FMH va négocier, outre les modèles de coûts, également la nomenclature avec les partenaires tarifaires curafutura, $\mathrm{H}+$ et CTM. Pour ce faire, les experts de la FMH sont déjà en contact avec l'ats-tms SA. Les négociations sur les positions du domaine "prestations de base médicales et non médicales» et sur des sujets généraux tels que les positions «rapports et résultats d'examen» sont en cours depuis un certain temps déjà au sein de l'organisation commune. D'autre part, les négociations au sujet de la nomenclature ont été entamées à la fin de l'année dernière. La FMH et les experts médicaux des sociétés de discipline vont inclure la nomenclature TARCO dans les négociations. Parallèlement, les assureurs feront part de leurs revendications (par ex. limitations).

La FMH maintient son objectif de soumettre, avec les partenaires tarifaires, la structure tarifaire révisée le 30 juin 2018 à l'approbation du Conseil fédéral. Au terme des négociations fructueuses, la structure devra d'abord être approuvée au printemps 2018 par l'Assemblée des délégués de la FMH et ensuite par la Chambre médicale.

\section{TARCO, la seule alternative à un tarif étatique}

Pour la FMH, la révision globale du TARMED, de concert avec toutes les organisations de médecins et les partenaires tarifaires, est la seule voie à suivre. Après l'intervention tarifaire du Conseil fédéral, il est impératif de mettre en vigueur une structure tarifaire appropriée et conforme aux règles de l'économie d'entreprise. 